ISSN: 1679-3013

D.O.I.: 10.5914/1679-3013.0061

\title{
BIOMASSA FITOPLANCTÔNICA E FATORES AMBIENTAIS EM UM ESTUÁRIO TROPICAL DO BRASIL.
}

Recebido em: 29/11/2011

Aceito em: 28/02/2012

\author{
Eveline Pinheiro de AQUINO ${ }^{1}$ \\ Lucas Guedes Pereira FIGUEIRÊDO ${ }^{1}$ \\ Diego Lira dos ANJOS ${ }^{1}$ \\ José Zanon de Oliveira PASSAVANTE ${ }^{1,2}$ \\ Maria da Glória Gonçalves da SILVA-CUNHA ${ }^{1,2}$
}

\section{RESUMO}

Os estudos acerca das características que regulam a biomassa fitoplanctônica objetivam a compreensão das mudanças do ambiente em escalas temporal e espacial, sendo este o foco principal do trabalho. As coletas foram realizadas em frequência mensal, em quatro pontos ao longo do estuário do rio dos Passos, litoral sul de Pernambuco, Brasil, no período de agosto/2009 a julho/2010. Foram obtidos os dados referentes à pluviosidade, profundidade, transparência, temperatura e amostras da água com auxílio de garrafa de Kitahara para medidas de salinidade e clorofila a. Os dados foram tratados através de análise de variância e componentes principais. O ambiente apresenta homogeneidade vertical térmica e salina, bem como pouca profundidade local. A salinidade e transparência da água estão em relação inversa à clorofila $a$, sendo estes os fatores possivelmente condicionantes da biomassa algal, necessitando de estudos futuros para ser analisada essa variável em função de outros fatores físicos, químicos e biológicos não abordados neste estudo.

Palavras chave: biomassa algal, fitoplâncton e dinâmica estuarina.

\section{ABSTRACT}

The studies about the features that regulate phytoplankton biomass aim to understand the environmental changes in temporal and spatial scales, which is the main focus of this work. Samples were collected monthly at four points along the Passos River Estuary, Pernambuco, Brazil, from August/2009 to July/2010. Were obtained data of rainfall, depth, transparency, temperature and water samples using Kitahara bottle for measure salinity and chlorophyll $a$. The data were analyzed using analysis variance and principal component analysis. The environment is homogeneous vertical thermal and saline and is shallow. The salinity and water transparency are in inverse relation to chlorophyll $a$, which are the factors possibly affecting the algal biomass, requiring further studies to analyze this variable with another physical, chemical and biological not covered in this study.

Key words: algal biomass, phytoplankton and dynamic estuarine.

\section{INTRODUÇÃO}

Estuários podem ser considerados ecossistemas de transição e por isso apresentam mudanças relevantes na composição, biomassa e produtividade das comunidades biológicas, sobretudo as fitoplanctônicas (MUYLAERT et al., 2009), as quais sofrem consequências da oscilação das características ambientais (CLOERN; JASSBY, 2008).

1 - Universidade Federal de Pernambuco/Programa de Pós-Graduação em Oceanografia

2 - Departamento de Oceanografia/Universidade Federal de Pernambuco 
AQUINO, Eveline Pinheiro de et al. Biomassa fitoplanctônica e fatores ambientais em um estuário tropical do Brasil.

Dessa forma, o entendimento dos fatores que regulam o fitoplâncton e sua produção primária tem sido o foco principal de vários estudos em ecossistemas estuarinos das regiões temperadas (ALMEIDA et al., 2002; BOYER et al., 2009; ROCHELLE-NEWALL et al., 2011) e tropicais (SANTIAGO et al., 2005; AZEVEDO et al., 2008; SANTOS et al., 2009), de forma a contribuir para a abrangência das mudanças do ambiente em escalas temporal e espacial, sendo ainda essenciais em estudos preditivos que objetivam a manutenção da qualidade dos ecossistemas aquáticos (CLOERN; JASSBY, op. cit.).

Estudos acerca dos organismos fitoplanctônicos em estuários apontam elevadas concentrações de clorofila a em diferentes períodos climáticos ao longo do ano (SIN et al., 2000; BRANCO et al., 2002; VAN DER MOLEN; PRESSINOTTO 2011), sendo portanto a oscilação na precipitação de chuvas o fator que causa maiores efeitos na biomassa algal (PASSAVANTE; FEITOSA, 2004).

Outras características ambientais, em função não só da sazonalidade, mas também da espacialidade horizontal, aparecem como importantes variáveis que afetam a distribuição e concentração da clorofila $a$, como a turbidez e a transparência da água (SHEN et al., 2011), a salinidade (DAMME et al., 2005; MATTA; FLYNN, 2008; KASAI et al., 2010), a temperatura (THOMPSON et al., 2008), dentre outros.

Por se tratar de um ambiente fortemente influenciado pela entrada de águas marinhas e com uma área de manguezal em bom estado de conservação (CPRH, 1999), o estuário do rio dos Passos, localizado no litoral sul do Estado de Pernambuco, Brasil, foi o local de desenvolvimento desta pesquisa.

Diante do exposto, o trabalho teve como objetivo identificar a variação da biomassa algal em função das características espacialidade horizontal, pluviosidade, profundidade local, transparência, temperatura e salinidade da água. Espera-se que as variáveis hidrológicas, principalmente transparência e salinidade, sejam afetadas pela dinâmica temporal e consequentemente causem efeitos na distribuição e concentração da biomassa fitoplanctônica.

\section{ÁREA DE ESTUDO}

O rio dos Passos, inserido na Área de Proteção Ambiental de Guadalupe, está localizado no litoral sul do Estado de Pernambuco, no município de Rio Formoso e distante aproximadamente $92 \mathrm{~km}$ da capital, Recife. O mesmo compõe o complexo estuarino do rio Formoso, juntamente com este rio e o Ariquindá, os quais sofrem forte influência marinha (mesomarés), estando o rio dos Passos localizado na zona estuarina média $\left(8^{\circ} 37^{\prime} 53,67^{\prime \prime S}\right.$ e $35^{\circ} 5^{\prime} 3,81^{\prime \prime} \mathrm{W} ; 8^{\circ} 40^{\prime} 50,50$ "S e $\left.35^{\circ} 6^{\prime} 46,44^{\prime \prime} \mathrm{W}\right)$, no limite até a sua bifurcação com o rio Formoso (Fig. 1). Este complexo estuarino é ainda formado pelos rios Lemenho e Porto das Pedras, os quais são afluentes do rio dos Passos (CPRH, op. cit.).

A Área de Proteção Ambiental (APA) de Guadalupe foi criada pelo Decreto n¹9.635 de 13 de março de 1997, que teve o intuito de preservar os ecossistemas localizados no entorno dos rios, principalmente manguezais, remanescentes de mata Atlântica, mata de restinga e cordões de recifes. Tais ambientes têm sido atingidos por atividades antrópicas, como a expansão imobiliária desordenada, a prática de queimadas e corte de mangue para expansão de áreas de cultivo, bem como a retirada indiscriminada de madeira e lenha, o que acarreta em desproteção das nascentes, exposição dos solos à erosão e consequentemente, o assoreamento dos rios e reservatórios da área (CPRH, 1999).

Com intuito de melhor conhecer essas áreas e para possíveis planos de conservação ambiental, algumas questões foram alvos de estudos ao longo da APA de Guadalupe, com abordagens acerca da meiofauna (VASCONCELOS et al., 2004), a biomassa e os organismos fitoplanctônicos (HONORATO-DA-SILVA et al., 2004; 2009; GREGO et al., 2009), a área de restinga (SILVA et al., 2008), a avifauna (RODRIGUES et al., 2007), a ocorrência de fungos (DAMASCENO et al., 2009), dentre outros. 
AQUINO, Eveline Pinheiro de et al. Biomassa fitoplanctônica e fatores ambientais em um estuário tropical do Brasil.

O clima da região é tropical quente e úmido, com temperaturas médias anuais em torno de $25,2^{\circ} \mathrm{C}$ e pluviosidade média entre 1500 a mais de $2000 \mathrm{~mm}$. Apresenta variação entre duas estações, estiagem e chuvosa, esta última abrangendo os meses de março a agosto, sendo julho o mês mais frio e chuvoso, enquanto que o período de estiagem compreende os meses de setembro a fevereiro, sendo novembro e dezembro os meses mais quentes (ITEP, 2010).

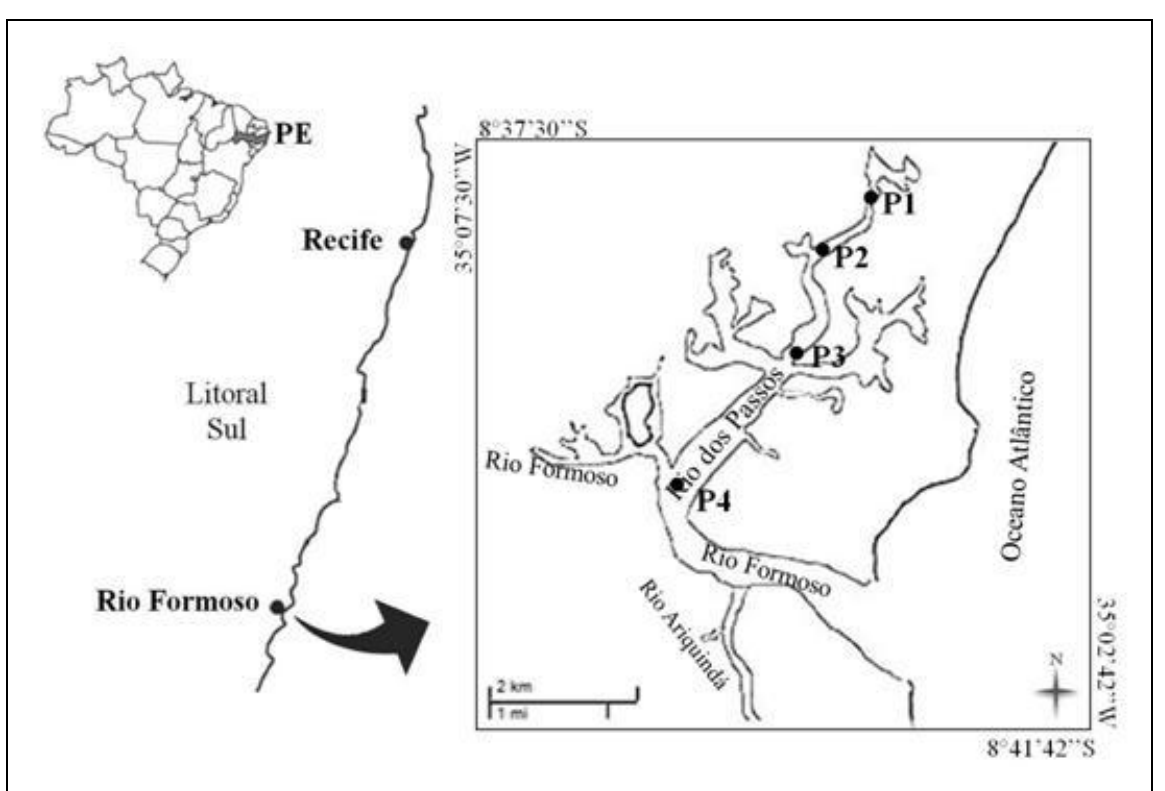

Figura 1 - Área estuarina do rio dos Passos, no estado do Pernambuco, Brasil, onde estão localizados os pontos de coleta P1, P2, P3 e P4 (Fonte: Google Earth, 2010).

\section{MATERIAL E MÉTODOS}

\section{Coleta dos dados}

Foram delimitados quatro pontos com auxílio de GPS, para coletas mensais, no período de agosto de 2009 a julho de 2010, abrangendo os períodos de estiagem e chuvoso, nas baixa-mares, em maré de sizígia.

A altura das marés foi obtida da Tábua de Marés, do Banco Nacional de Dados Oceanográficos da Diretoria de Hidrografia e Navegação (DHN) da Marinha do Brasil, referentes ao Porto de Suape, Pernambuco.

Os dados referentes à precipitação de chuvas para a área em estudo foram obtidos do Laboratório de Meteorologia do Instituto de Tecnologia de Pernambuco (LAMEPE/ITEP), referente à Estação de Cucau ( $8^{\circ} 40^{\prime} 18^{\prime \prime} \mathrm{S}$ e $\left.35^{\circ} 11^{\prime} 24^{\prime \prime} \mathrm{W}\right)$, localizada no município de Rio Formoso e correspondente à precipitação de chuvas mensal (agosto de 2009 a julho de 2010) e a normal climatológica (últimos 30 anos).

A profundidade local foi obtida com uso de uma sonda manual, acoplada a um cabo graduado $(\mathrm{cm})$ e a partir da profundidade do disco de Secchi foi determinada a transparência da água. As amostras de água foram coletadas com uso de uma garrafa de Kitahara e os dados de temperatura e salinidade (superficial e fundo), obtidos in situ com uso de um termômetro digital e refratômetro, respectivamente. As amostras correspondentes à clorofila a foram acondicionadas em frascos de polietileno fosco (1L) para posterior filtragem no Laboratório de Fitoplâncton do Departamento de Oceanografia da Universidade Federal de Pernambuco. Dessa forma, a biomassa $\left(\mathrm{mg} . \mathrm{m}^{-}\right.$ ${ }^{3}$ ) foi determinada a partir da concentração de clorofila $a$, com filtragem e extração dos pigmentos utilizando sistema de filtros Milipore ${ }^{\circledR} \mathrm{HA}(0,47 \mathrm{~mm}$ de diâmetro de $0,45 \mu \mathrm{m}$ de porosidade), bem como o uso de acetona a $90 \%$, com a posterior análise espectrofotométrica segundo Strickland e Parsons (1963), descrita pela UNESCO (1966).

\section{Análises estatísticas}


AQUINO, Eveline Pinheiro de et al. Biomassa fitoplanctônica e fatores ambientais em um estuário tropical do Brasil.

Para verificar a normalidade dos dados foi utilizado o teste de Shapiro-Wilk. As variações espacial, sazonal e a interação destas nas características hidrológicas (profundidade local, temperatura e salinidade superficial e fundo, transparência da água e biomassa fitoplanctônica) foram testadas através de ANOVA fatorial, com teste a posteriori de Tukey. A homogeneidade das variâncias foi verificada a partir do teste de Bartlett. Todas essas análises foram realizadas no programa Statistica 7.0 (STATSOFT, 2004). Foi realizada a Análise dos Componentes Principais (ACP) com extração do autovalor e autovetor. Para elaboração desta foi utilizado o programa NTSYSpc 2.21 (LEGENDRE; LEGENDRE，1984).

\section{RESULTADOS}

\section{Pluviosidade}

Durante o período em estudo, o total de chuvas precipitado foi de $1865,9 \mathrm{~mm}$. A sazonalidade foi marcante, com distinção dos períodos chuvoso (março a agosto) e de estiagem (setembro a fevereiro), com valor mínimo de $22 \mathrm{~mm}$ em novembro de 2009 e um pico de chuvas de 592,5 mm alcançado em junho de 2010 (Fig. 2).

\section{Figura 2 - Pluviosidade $(\mathrm{mm})$, indicando a normal climatológica de chuvas, correspondente aos últimos 30 anos (1980-2010) e a média mensal, referente ao período de coletas (agosto de 2009 a julho de 2010) (Fonte: ITEP, 2010).}

\section{Profundidade local}

A profundidade média apresentou diferença significativa entre os pontos de coleta $(F=57,42 ; p<0,01)$, variando de $1,80 \mathrm{~m}$, no ponto $\mathrm{P} 2$ a $4,60 \mathrm{~m}$ no ponto $\mathrm{P} 3$, sendo este o ponto mais profundo, com média de $4,07 \mathrm{~m}$, enquanto que 0 ponto $\mathrm{P} 2$ pode ser considerado ligeiramente mais raso, com média de 2,26 m.

Não houve diferença sazonal significativa $(F=0,01 ; p=0,96)$, com os valores mínimo e máximo registrados na estação chuvosa, como observado na Tabela 1, a qual mostra os principais valores de todas as variáveis estudadas.

Porém, o teste de Tukey aposteriori mostrou que o efeito sazonal acentuou a diferença espacial, em que o ponto $\mathrm{P} 4$, durante a estiagem, apresentou diferença significativa entre o ponto P2 na estiagem e na chuva ( $p<0,01$ para ambos) (Fig. 3.A).

\section{Transparência da água}

O ponto próximo a montante ( $\mathrm{P} 1)$ apresentou menor média de transparência da água, com $70 \mathrm{~cm}$ durante o período de maiores chuvas e maior média de $2,0 \mathrm{~m}$ no ponto P4, durante a estiagem (Fig. 3.B). Houve diferença espacial significativa ( $F=30,27 ; p<$ $0,01)$, com gradiente crescente no sentido ao ponto de maior influência marinha (P4).

As maiores profundidades de Secchi foram durante a estiagem, com diferença sazonal significativa $(F=12,09 ; p<0,01)$, e ambos os valores mínimo e máximo registrados no período chuvoso (tab. 1 ). 
AQUINO, Eveline Pinheiro de et al. Biomassa fitoplanctônica e fatores ambientais em um estuário tropical do Brasil.

Tabela 1 - Variação dos fatores ambientais no estuário do rio dos Passos, correspondentes aos valores mínimos, máximos e médios, nos diferentes períodos climáticos (chuvoso e estiagem).

\begin{tabular}{lrrr|rrr}
\hline \multicolumn{1}{c}{ Variáveis } & \multicolumn{3}{c}{ Estiagem } & \multicolumn{3}{c}{ Chuvoso } \\
& Mín & Máx & Média & Mín & Máx & Média \\
\hline Normal climatológica de chuvas (mm) & 0,2 & 407,6 & 126,6 & 23,3 & 800,5 & 248,3 \\
Pluviosidade 2009-2010 (mm) & 22,0 & 145,7 & 75,3 & 79,5 & 592,5 & 235,3 \\
Profundidade (m) & 2,1 & 4,5 & 2,9 & 1,8 & 4,6 & 2,9 \\
Transparência (m) & 0,8 & 2,3 & 1,6 & 0,4 & 2,4 & 1,4 \\
Temperatura superficial $\left({ }^{\circ} \mathrm{C}\right)$ & 27,2 & 31,1 & 28,9 & 23,0 & 29,5 & 27,4 \\
Temperatura profunda $\left({ }^{\circ} \mathrm{C}\right)$ & 27,2 & 31,0 & 28,7 & 24,0 & 29,0 & 27,4 \\
Salinidade (superfície) & 20,0 & 35,0 & 29,5 & 14,0 & 30,0 & 24,2 \\
Salinidade (fundo) & 24,0 & 35,0 & 30,5 & 13,0 & 30,0 & 23,8 \\
Clorofila a (mg.m ${ }^{-3}$ ) & 1,2 & 20,9 & 7,61 & 2,0 & 21,3 & 6,25 \\
\hline
\end{tabular}

\section{Temperatura}

Não houve diferença espacial horizontal e vertical significativa na temperatura da água $(F=0,31 ; p=0,81 ; F=0,19 ; p=0,90$, respectivamente) (Fig. 3.C e 3.D). Porém, foi observado um gradiente decrescente dos valores, no sentido próximo a jusante. A menor média registrada foi de $27,2^{\circ} \mathrm{C}$ no período chuvoso e no ponto intermediário $\mathrm{P} 2$, sendo a maior de $29,4^{\circ} \mathrm{C}$ na estiagem e no ponto mais interno (P1). Portanto, houve diferença significativa entre as estações climáticas $(F=13,21 ; p<0,01)$ com maiores valores na estiagem.

\section{Salinidade}

A salinidade superficial da água apresentou diferença espacial significativa ( $F=$ $3,73 ; p=0,01)$, enquanto que na camada mais próxima ao sedimento não houve essa diferença $(F=1,77 ; p=0,16)$ ( $F i g .3 . E$ e 3.F) e a coluna d'água esteve ausente de estratificação salina, com os valores variando de 13 a 35 (tab. 1). Mas, ambas as profundidades estiveram com os valores distribuídos em um gradiente crescente no sentido a jusante, com a camada superficial apresentando a menor média, no ponto mais a montante (P1), durante o período chuvoso, correspondente a 21,3 e a maior de 31,3 no ponto $\mathrm{P} 4$, durante o período de estiagem. A diferença sazonal foi significativa para esta variável $(F=22,73 ; p<0,01)$, com maiores teores na estiagem.

\section{Biomassa fitoplanctônica}

Os valores de biomassa oscilaram de $1,2 \mathrm{mg} \cdot \mathrm{m}^{-3}$ a $21,3 \mathrm{mg} \cdot \mathrm{m}^{-3}$ (tab.1). A média mínima foi encontrada no ponto de maior influência marinha (P4), durante a estiagem, correspondente a $4,19 \mathrm{mg} \cdot \mathrm{m}^{-3}$, enquanto que a maior foi de $10,58 \mathrm{mg} \cdot \mathrm{m}^{-3}$ registrada no ponto P1 e na estação chuvosa (Fig. 3.G). Não houve diferenças significativas espacialmente $(F=2,75 ; p=0,05)$ ou sazonalmente $(F=0,97 ; p=0,32)$. Porém, pode ser observado um gradiente crescente em direção à porção mais a montante do estuário.

\section{Análise dos componentes principais (ACP)}

Foi possível observar que a primeira componente explicou $38,2 \%$ dos dados, em que houve correlação inversa da biomassa fitoplanctônica $(r=0,528)$ com a transparência da água $(r=0,809)$ e salinidade $(r=0,803)$, enquanto que a segunda 
AQUINO, Eveline Pinheiro de et al. Biomassa fitoplanctônica e fatores ambientais em um estuário tropical do Brasil.

componente explicou $23,4 \%$ dos dados, com a temperatura da água $(r=0,671)$ inversa à pluviosidade ( $r=0,595)$ (tab. 2).

Tabela 2 - Variação dos dados e coeficientes de correlação entre as variáveis analisadas, das duas primeiras componentes da $\mathrm{ACP}$, no estuário do rio dos Passos, Brasil.

\section{Componente 1 Componente 2}

Variação dos dados (\%)

$$
38,2 \%
$$$$
23,4 \%
$$

Temperatura superficial

0,4914

0,6714

\section{Salinidade superficial}

0,8039

0,1754

Transparência da água

0,8093

$-0,3396$

Profundidade

0,4866

$-0,4537$

\section{Biomassa}

$-0,5285$

0,4966

Pluviosidade

$-0,4823$

$-0,5954$
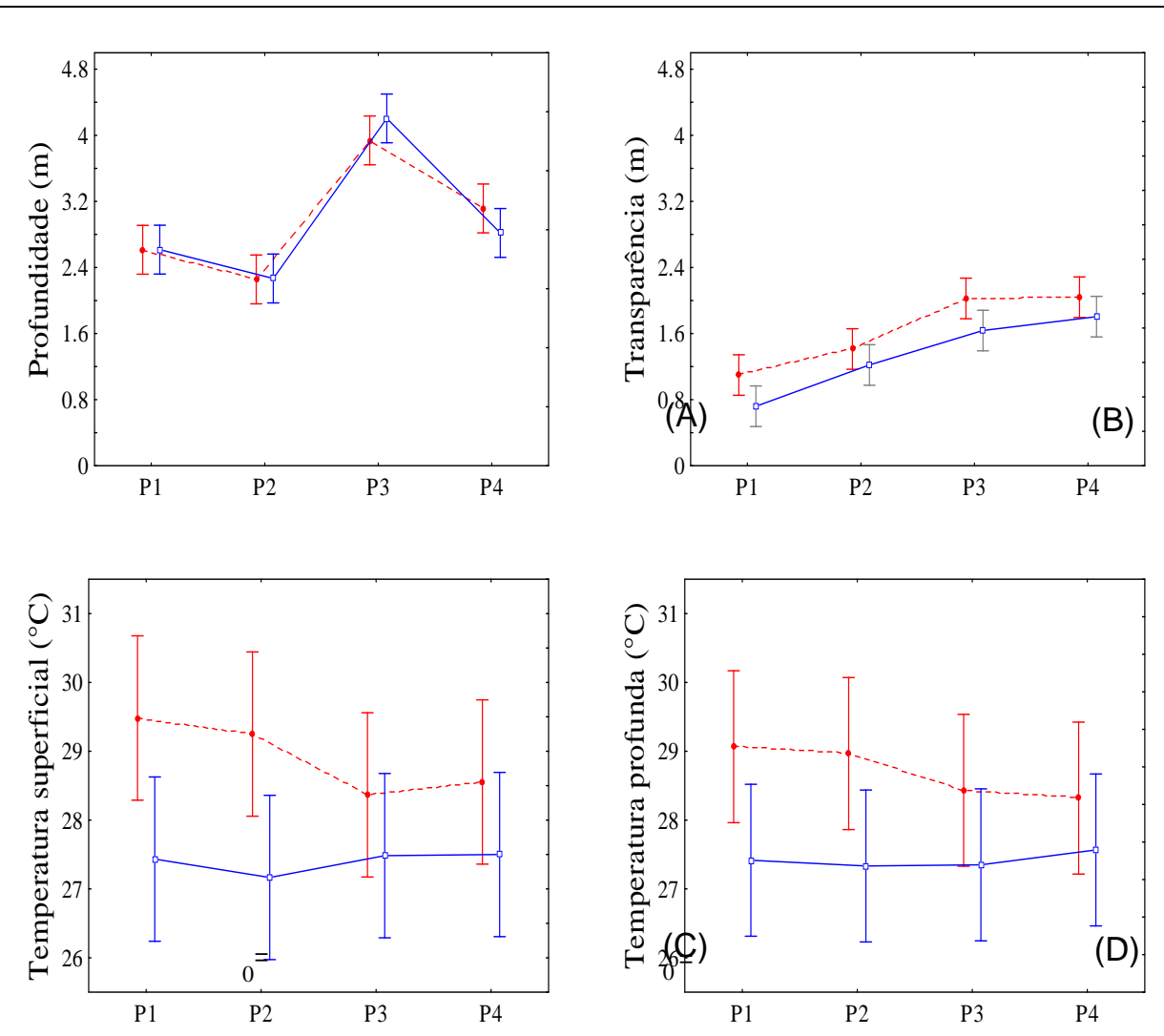
AQUINO, Eveline Pinheiro de et al. Biomassa fitoplanctônica e fatores ambientais em um estuário tropical do Brasil.

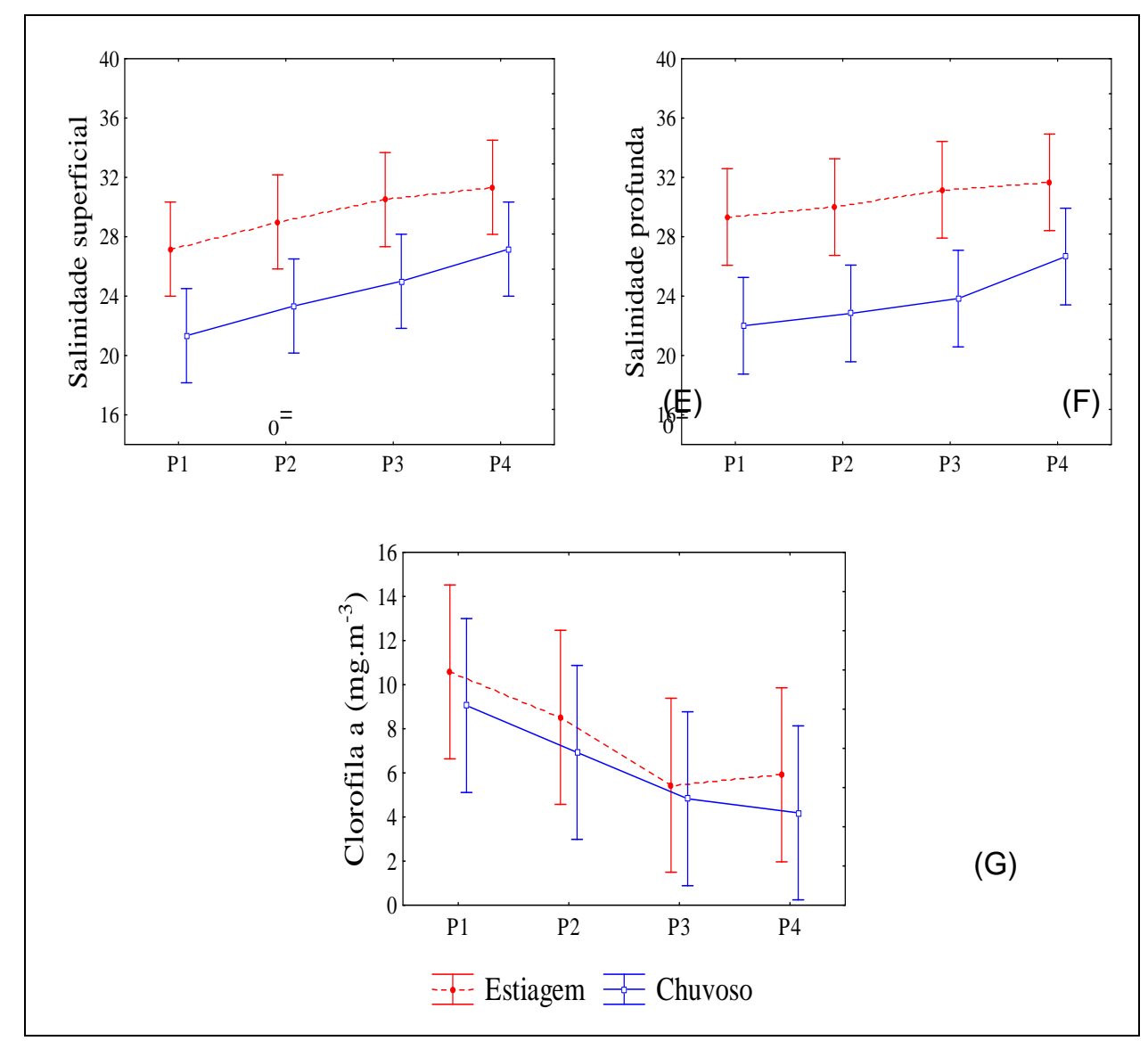

Figura 3 - Variação espacial e sazonal das médias das características hidrológicas do estuário do rio dos Passos: (A) Profundidade local - $\mathrm{m}$; (B) Transparência da água - $\mathrm{m}$; (C) Temperatura da água superficial e (D) profunda $-{ }^{\circ} \mathrm{C}$; (E) Salinidade superficial e (F) profunda; (G) Clorofila $a-m g \cdot \mathrm{m}^{-3}$.

\section{DISCUSSÃO}

As características hidrológicas do estuário do rio dos Passos estão controladas pela precipitação de chuvas e esta condição, de acordo com Cloern e Jassby (2008), reflete na manutenção da qualidade da água. A pluviosidade está correlacionada com o grau de influência terrígena e a magnitude da transparência da água, existindo, segundo Passavante e Feitosa (2004), dois padrões de comportamento anual da biomassa fitoplanctônica para as áreas costeiras do nordeste do Brasil. Assim, durante o período de maiores chuvas há um incremento algal, como registrado, por exemplo, por Leão et al. (2008) para o estuário do rio Igarassu e Honorato-da-Silva et al. (2009) no estuário do rio Formoso (ambos em Pernambuco, Brasil), em função do aporte de nutrientes de origem terrígena e quando a disponibilidade de luz não é fator limitante.

Entretanto, o período de estiagem pode também estar associado a um maior desenvolvimento destes organismos, justificado pela profundidade da camada fótica e turbidez da água (AZEVEDO et al., 2008), ou mesmo pela direção dos ventos, o fluxo fluvial (FUJITA; ODEBRECHT, 2007), o regime de marés e as características hidrográficas do rio (GREGO et al., 2009), sendo este último fator o que possivelmente explica a condição de que a biomassa algal do estuário em estudo não sofreu diferença sazonal significativa, sendo considerado um rio litorâneo e de pequena bacia hidrográfica.

As regiões estuarinas são, em geral, ambientes considerados rasos (SIN et al., 2000; COSTA et al., 2008; ABREU et al., 2010; VAN DER MOLEN; PRESSINOTO, 2011). A tabela 3 mostra a variação de profundidade, bem como das demais variáveis, em alguns estuários do Estado do Pernambuco, Brasil. É observado, portanto, que nas regiões 
AQUINO, Eveline Pinheiro de et al. Biomassa fitoplanctônica e fatores ambientais em um estuário tropical do Brasil.

próximas ao rio dos Passos predominam estuários pouco profundos. Esta variável sofre efeitos da topografia do sedimento (BASTOS et al., 2011), como observado para o local em estudo.

A baixa profundidade está relacionada à proximidade da camada de água superficial ao sedimento, o que propicia ressuspensão de células fitoplanctônicas e nutrientes depositados no fundo e um incremento na biomassa algal (DAMME et al., 2005).

A transparência da água é uma variável física dependente da precipitação de chuvas, como acontece para alguns estuários brasileiros estudados por Bastos et al. (op. cit.) (estuário do rio Maracaípe, Pernambuco) e por Masuda et al. (2011) (sistema estuarino de Santos, São Paulo), bem como da topografia local, como observado no estuário do rio dos Passos e manifesta-se em um gradiente crescente em direção à porção de maior influência marinha (BRANCO et al., 2002; GREGO et al., op. cit.).

Quanto à oscilação sazonal, a disponibilidade de luz pode limitar o crescimento algal durante o período chuvoso (DAMME et al., op. cit.; GAMEIRO et al., 2011) ou não (HONORATO-DA-SILVA et al., 2004), dependendo da intensificação da lixiviação terrestre e, consequentemente, maiores quantidades de material em suspensão estará disponível na coluna d'água, dificultando a penetração de luz (ESKINAZI-LEÇA et al, 2004). O contrário acontece para o período de estiagem (AZEVEDO et al., 2008), como registrado para a presente pesquisa, com maiores concentrações da biomassa algal nestes meses mais quentes e em uma relação inversa à disponibilidade de luz.

Tabela 3 - Variação (mínimo-máximo) dos fatores profundidade, temperatura da água, salinidade, transparência e biomassa fitoplanctônica para os estuários do litoral sul do Estado de Pernambuco, Brasil.

\begin{tabular}{|c|c|c|c|c|c|c|}
\hline Referência & $\begin{array}{l}\text { Área } \\
\text { estuarina }\end{array}$ & Prof(m) & $\operatorname{Temp}\left({ }^{\circ} \mathrm{C}\right)$ & Sal & Trans(m) & $\begin{array}{l}\text { Biomassa } \\
\left(\mathrm{mg}^{\left.-\mathrm{m}^{-3}\right)}\right.\end{array}$ \\
\hline $\begin{array}{l}\text { Presente } \\
\text { estudo }\end{array}$ & $\begin{array}{l}\text { Rio dos } \\
\text { Passos }\end{array}$ & $1,8-4,6$ & $23-29,5$ & $13-35$ & $0,4-2,4$ & $1,2-20,9$ \\
\hline $\begin{array}{l}\text { Branco et } \\
\text { al. (2002) }\end{array}$ & $\begin{array}{l}\text { Barra das } \\
\text { Jangadas }\end{array}$ & $1,2-6,0$ & $25-30,5$ & $0-38$ & $0,3-2,4$ & $0,57-49,84$ \\
\hline $\begin{array}{l}\text { Honorato- } \\
\text { da-Silva et } \\
\text { al. }(2004)\end{array}$ & $\begin{array}{l}\text { Rio } \\
\text { Formoso }\end{array}$ & $1,8-9,7$ & $24,5-29,5$ & $\begin{array}{l}1,3- \\
36,3\end{array}$ & $0,25-3,6$ & $2,43-70,22$ \\
\hline $\begin{array}{l}\text { Bastos et } \\
\text { al. (2005) }\end{array}$ & Rio Una & - & $23,8-32$ & $0-39$ & $0,2-2,25$ & $1,68-36,3$ \\
\hline $\begin{array}{l}\text { Noriega et } \\
\text { al. (2009) }\end{array}$ & $\begin{array}{l}\text { Barra das } \\
\text { Jangadas }\end{array}$ & $1,4-5,2$ & $25,2-30,4$ & $\begin{array}{r}0,2- \\
35,5\end{array}$ & $0,4-1,4$ & $7,76-158,6$ \\
\hline $\begin{array}{l}\text { Grego et al. } \\
(2009)\end{array}$ & $\begin{array}{l}\text { Rio } \\
\text { Ariquindá }\end{array}$ & - & $25-31,5$ & $\begin{array}{l}3,4- \\
37,9\end{array}$ & $0,20-4$ & $1,09-18,21$ \\
\hline $\begin{array}{l}\text { Bastos et } \\
\text { al. (2011) }\end{array}$ & $\begin{array}{l}\text { Rio } \\
\text { Maracaípe }\end{array}$ & $0,25-2,7$ & $26-31,5$ & $1-37$ & $0,25-2,7$ & $1,11-18,72$ \\
\hline
\end{tabular}

A temperatura da água em estuários subtropicais e tropicais é de pequena oscilação (tab. 3) e influencia a distribuição e concentração do fitoplâncton quando em combinação com outras variáveis (PASSAVANTE; FEITOSA, 2004), diferentemente para regiões estuarinas temperadas (SIN et al., 2000; GAMIERO et al., 2011), que estão sujeitas a amplas oscilações de temperatura, com o padrão sazonal de florescimento do fitoplâncton no decorrer das estações do ano. No estuário do rio dos Passos foi possível inferir que a temperatura da água não apresentou correlação com a biomassa algal, bem como não sofreu diferença espacial, somente sazonal, estando em uma relação inversa à pluviosidade. Quanto ao padrão vertical, o estuário pode ser classificado como homogêneo termicamente. 
AQUINO, Eveline Pinheiro de et al. Biomassa fitoplanctônica e fatores ambientais em um estuário tropical do Brasil.

A estratificação também foi ausente quanto aos teores de salinidade e o estuário foi classificado como mesoalino a eualino. A salinidade pode ser considerada um dos fatores principais para a oscilação da biomassa, com uma relação inversamente proporcional destas variáveis, como registrado para o local em estudo e outros estuários (ALMEIDA et al., 2002; LOSADA et al., 2003).

A concentração da clorofila a tem sido amplamente utilizada como um indicador da biomassa fitoplanctônica (CLOERN; JASSBY, 2010; GAMEIRO; BROTAS, 2010). Em águas estuarinas tropicais, a precipitação de chuvas parece ser a variável de maior influência para o fitoplâncton (PASSAVANTE; FEITOSA, op. cit.) e a maior concentração de clorofila a pode acontecer no período chuvoso (SANTIAGO et al., 2005; BASTOS et al., 2011) ou em meses mais quentes (ALMEIDA et al., op. cit.; LOSADA et al., op. cit.; COSTA et al., 2008; AZEVEDO et al., 2008; CLOERN; JASSBY, 2008; GAMEIRO et al., 2011). Para o estuário do rio dos Passos, a biomassa não apresentou variação significativa entre os períodos climáticos (chuvoso e estiagem), porém os valores foram discretamente maiores durante a estiagem, fato este também descrito para o estuário do rio Ariquindá, por Grego et al. (2009).

As maiores concentrações de clorofila a podem ser registradas na porção superior dos estuários (DAMME et al., 2005). Quando comparado o padrão de distribuição espacial horizontal da biomassa fitoplanctônica no complexo estuarino do rio Formoso, onde está inserido o rio dos Passos, juntamente com o rio Ariquindá, é observado que as maiores concentrações de clorofila $a$ encontram-se nas zonas próximas a montante (HONORATODA-SILVA et al., 2004; GREGO et al., 2009), e portanto, sob influência de menores teores de salinidade, atestando a relação inversa desta variável com a biomassa fitoplanctônica do estuário do rio dos Passos.

Para os estuários próximos ao local em estudo (tab. 3), Branco et al. (2002) e Noriega et al. (2009), classificam a zona estuarina de Barra das Jangadas como eutrófico e que sofre forte ação antrópica. O estuário do rio Una, por sua vez, pode ser classificado como bastante produtivo e eutrófico (BASTOS et al., 2005), enquanto que a zona estuarina de Maracaípe é mesotrófica e está sem influência de poluentes (BASTOS et al., 2011). Por fim, quando analisados os estudos no complexo estuarino do rio Formoso, Grego et al. (op. cit.) afirmam que o rio Ariquindá apresenta moderada produção, estando o ambiente livre de fortes impactos antrópicos, enquanto que Honorato-da-Silva et al. (2004) considera o estuário do rio Formoso como sendo eutrófico e de excelente suporte aos níveis tróficos do ecossistema. Dessa forma, quando comparados os teores de clorofila a desses ecossistemas, é possível observar que os valores de biomassa do estuário do rio dos Passos indicam um ambiente de características eutróficas e em bom estado de conservação.

O conjunto de dados do estuário do rio dos Passos apresentados poderá contribuir para análises de padrões e dinâmica da biomassa fitoplanctônica, em resposta à oscilação da precipitação de chuvas, profundidade local, temperatura, salinidade e transparência da água, bem como a dinâmica dessas variáveis hidrológicas em estuários tropicais rasos.

\section{CONCLUSÕES}

1. A porção estuarina do rio dos Passos é pouco profunda, de águas transparentes, temperatura, salinidade e biomassa fitoplanctônica elevadas, esta não apresentando efeito da oscilação sazonal e espacial, uma vez que trata-se de um rio litorâneo e de pequena bacia hidrográfica;

2. o estuário em estudo é do tipo bem misturado, uma vez que apresenta homogeneidade térmica e salina da coluna d'água;

3. a clorofila a está relacionada inversamente à salinidade e transparência da água, sendo estes os fatores condicionantes da concentração da biomassa fitoplanctônica, necessitando de estudos futuros para ser analisada essa variável em função de outros fatores físicos, químicos e biológicos, não abordados neste estudo. 
AQUINO, Eveline Pinheiro de et al. Biomassa fitoplanctônica e fatores ambientais em um estuário tropical do Brasil.

\section{AGRADECIMENTOS}

Os autores agradecem ao Conselho Nacional de Desenvolvimento Científico e Tecnológico - CNPq, pelo apoio financeiro, Processo n 304992/2006-2; à Coordenação de Aperfeiçoamento de Pessoal de Nível Superior - CAPES, pela bolsa concedida ao primeiro autor; ao Programa de Pós-Graduação em Oceanografia da Universidade Federal de Pernambuco - UFPE, pela infra-estrutura.

\section{REFERÊNCIAS BIBLIOGRÁFICAS}

ABREU, P. C.; BERGESCH, M.; PROENÇA, L. A.; GARCIA, C. A. E.; ODEBRECHT, C. Shortand long-term chlorophyll a variability in the shallow microtidal Patos Lagoon Estuary, Southern Brazil. Estuaries and Coasts, v. 33, p. 554-569, 2010. DOI 10.1007/s12237009-9181-9

ALMEIDA, M. A.; CUNHA, M. A.; ALCÂNTARA, F. Seasonal change in the proportion of bacterial and phytoplankton production along a salinity gradient in a shallow estuary. Hydrobiologia, v. 475/476, p. 251-262, 2002.

AZEVEDO, A. C. G.; FEITOSA, F. A. N.; KOENING, M. L. Distribuição espacial e temporal da biomassa fitoplanctônica e variáveis ambientais no Golfão Maranhense, Brasil. Acta Botanica Brasilica, v. 22, n. 3, p. 870-877. 2008.

BASTOS, R. B.; FEITOSA, F. A. N.; MUNIZ, K. Variabilidade espaço-temporal da biomassa fitoplanctônica e hidrologia no estuário do rio Una (Pernambuco - Brasil). Tropical Oceanography, v. 33, n. 1, p. 1-18, 2005.

BASTOS, R. B.; FEITOSA, F. A. N.; KOENING, M. L.; MACHADO, R. C. A.; MUNIZ,K. Caracterização de uma zona costeira tropical (Ipojuca, Pernambuco - Brasil): produtividade fitoplanctônica e outras variáveis ambientais. Brazilian Journal of Aquatic Science and Technology, v. 15, n. 1, p. 01-10, 2011.

BOYER, J. N.; KELBLE, C. R.; ORTNER, P. B.; RUDNICK, D. T. Phytoplankton bloom status: Chlorophyll a biomass as an indicator of water quality condition in the southern estuaries of Florida, USA. Ecological Indicators, p. 56-67, 2009

BRANCO, E. S.; FEITOSA, F. A. N.; FLORES-MONTE, M. J. Variação sazonal e espacial da biomassa fitoplanctônica relacionada com parâmetros hidrológicos no estuário de Barra das Jangadas (Jaboatão dos Guararapes - Pernambuco - Brasil). Tropical

Oceanography, v. 30, n. 2, p. 79-96, 2002.

CLOERN, J. E.; JASSBY, A. D. Complex seasonal patterns of primary producers at the land-sea interface. Ecology Letters, v. 11, p. 1294-1303. 2008. DOI: 10.1111/j.14610248.2008.01244.x

CLOERN, J. E.; JASSBY, A. D. Patterns and Scales of Phytoplankton Variability in Estuarine-Coastal Ecosystems. Estuaries and Coasts, v. 33, p. 230-241, 2010. DOI 10.1007/s12237-009-9195-3

COSTA, L. S.; HUSSAR, V. L. M.; OVALLE, A. R. Phytoplankton functional groups in a tropical Estuary: hydrological control and nutrient limitation. Estuaries and Coasts, v. 32 , p. 508-521, 2008. DOI 10.1007/s12237-009-9142-3

CPRH - companhia pernambucana de controle da poluição ambiental e de administração de recursos hídricos. Diagnóstico sócio-ambiental e ZEEC do litoral sul de Pernambuco. Recife, 1999, 91p.

DAMASCENO, G.; COSTA, A. A. A.; PASSAVANTE, J. Z. O.; CAVALCANTI, L. H. Stemonaria fuscoides (Stemonitaceae, Myxomycetes): a new record for Brazil. Mycotaxon, v. 108, p. 205-211, 2009.

DAMME, V. D.; STRUYF, E.; MARIS, T.; YSEBAERT, T.; DEHAIRS, F.; TACKX, M.; HEIP, C.; MEIRE, P. Spatial and temporal patterns of water quality along the estuarine salinity gradient of the Scheldt estuary (Belgium and The Netherlands): results of an integrated 
AQUINO, Eveline Pinheiro de et al. Biomassa fitoplanctônica e fatores ambientais em um estuário tropical do Brasil.

monitoring approach. Hydrobiologia, v. 540, p. 29-45, 2005. DOI 10.1007/s10750004-7102-2

ESKINAZI-LEÇA, E.; KOENING, M.L. \& SILVA-CUNHA, M.G.G. Estrutura e dinâmica da comunidade fitoplanctônica. In: Eskinazi-Leça, E.; Newmann-Leitão, S.; Costa, M. F. (Ed.). Oceanografia: um cenário tropical. Recife: Edições Bagaço, 2004. p. 353-373

FUJITA, C. C.; ODEBRECHT, C. Short term variability of chlorophyll a and phytoplankton composition in a shallow area of the Patos Lagoon estuary (Southern Brazil). Atlântica, v. 29, n. 2, p. 93-106, 2007

GAMEIRO, C.; ZWOLINSKI, J.; BROTAS, V. Light control on phytoplankton production in a shallow and turbid estuarine system. Hydrobiologia, v. 669, p. 249-263, 2011. DOI 10.1007/s10750-011-0695-3

GAMEIRO, C.; BROTAS, V. Patterns of phytoplankton variability in the Tagus Estuary (Portugal). Estuaries and Coasts, v. 33, p. 311-323, 2010. DOI 10.1007/s12237-0099194-4.

GREGO, C. K. S.; FEITOSA, F. A. N.; HONORATO-DA-SILVA, M.; SILVA-CUNHA, M. G. G.; FILHO, G. A. N. Fitoplâncton do ecossistema estuarino do rio Ariquindá (Tamandaré, Pernambuco, Brasil): variáveis ambientais, biomassa e produtividade primária.

Atlântica, v. 31, n. 2, p. 183-198, 2009

HONORATO-DA-SILVA, M.; PASSAVANTE, J. Z. O.; SILVA-CUNHA, M. G. G.; NASCIMENTO-VIEIRA, D. A.; GREGO, C. K. S.; MUNIZ, K. Distribuição espacial e sazonal da biomassa fitoplanctônica e dos parâmetros hidrológicos no estuário do rio Formoso (Rio Formoso, Pernambuco, Brasil). Tropical Oceanography, v. 32, n. 1, p. 89-106, 2004.

ITEP - Instituto de Tecnologia De Pernambuco. Disponível em <http//:www.itep.br>. Acesso em 10 de março de 2010.

KASAI, A.; KURIKAWA, Y.; UENO, M.; ROBERT, D.; YAMASHITA, Y. Salt-wedge intrusion of seawater and its implication for phytoplankton dynamics in the Yura Estuary, Japan.

Estuarine, Coastal and Shelf Science, v. 86, p. 408-414, 2010.

LEÃO, B. M.; PASSAVANTE, J. Z. O. P.; SILVA-CUNHA, M. G. G.; Santiago, M. F. Ecologia do microfitoplâncton do estuário do rio Igarassu, PE, Brasil. Acta Botanica Brasilica. v. 22, n. 3, p. 711-722, 2008.

LEGENDRE, L.; LEGENDRE, P. Ecologie Numérique. 2. La structure des données écologiques. $2^{a}$ ed. Quebec: Masson Presses de L'Université du Quebec, 1984. 335p. (Collection d'Écologie, 13).

LOSADA, A. P. M.; FEITOSA, F. A. N.;LINS, I. C. Variação sazonal e espacial da biomassa fitoplanctônica nos estuários dos rios Ilhetas e Mamucaba (Tamandaré-PE) relacionada com parâmetros hidrológicos. Tropical Oceanography, v. 31, n. 1, p. 1-26, 2003.

MASUDA, L. S. M.; MOSER, G. A. O., BARRERA-ALBA, J. J. Variação temporal do fitoplâncton no canal estuarino de Santos (SP). Brazilian Joural of Aquatic Science and Technology, v. 15, n. 1, p. 79-93, 2011.

MATTA, M. E. M.; FLYNN, M. N. Estrutura da comunidade fitoplanctônica no gradiente de salinidade do estuário de Cananéia - SP. Revista Intertox de Toxicologia, Risco Ambiental e Sociedade, v. 1, n. 1, p. 59-69, 2008.

MUYLAERT, K.; SABBE, K.; VYVERMAN, W. Changes in phytoplankton diversity and community composition along the salinity gradient of the Schelde estuary (Belgium/The Netherlands). Estuarine, Coastal and Shelf Science, v. 82, p. 335-340, 2009.

NORIEGA, C. E.; MUNIZ, K.; FLORES-MONTES, M. J.; MACEDO, S. J.; ARAUJO, M.; FEITOSA, F. A. N.; LACERDA, S. R. Series temporales de variables hidrobiológicas em un estuario tropical (Brasil). Revista de Biología Marina y Oceanografía, v. 44, n. 1, p. 93-108, 2009. 
AQUINO, Eveline Pinheiro de et al. Biomassa fitoplanctônica e fatores ambientais em um estuário tropical do Brasil.

PASSAVANTE, J. Z. O.; FEITOSA, F. A. N. Dinâmica da produtividade fitoplanctônica na zona costeira marinha. In: Eskinazi-Leça, E.; Newmann-Leitão, S.; Costa, M. F. (Ed.). Oceanografia: um cenário tropical. Recife: Edições Bagaço, 2004. p. 425-440.

POOLE, H. H.; ATKINS, W. R. G. Photoeletric measurements of submarine illumination troughout the year. Journal Marine Biological Association United Kingdom, v. 16, p. 297-325, 1929.

RODRIGUES, R. C.; ARAÚJO, H. F. P.; LYRA-NEVES, R. M.; TELINO-JÚNIOR, W. R.; BOTELHO, M. C. N. Caracterização da Avifauna na Área de Proteção Ambiental de Guadalupe, Pernambuco. Ornithologia, v. 2, n. 1, p.47-61, 2007.

ROCHELLE-NEWALL, E. J.; CHU, V. T.; PRINGAULT, O.; AMOUROUX, D.; ARFI, R.; BETTAREL, Y.; BOUVIER, T.; BOUVIER, C.; GOT, P.; NGUYEN, T. M. H.; MARI, X.; P. NAVARRO3, DUONG, T. N.; CAO, T. T. T.; PHAM, T. T.; OUILLON, S.; TORRÉTON, J. P. Phytoplankton diversity and productivity in a highly turbid, tropical coastal system (Bach Dang Estuary, Vietnam). Biogeosciences Discuss, v. 8, p. 487-525, 2011

SANTIAGO, M. F.; PASSAVANTE, J. Z. O.; SILVA-CUNHA, M. G. G. Caracterização de parâmetros físicos, químicos e biológico em ambiente hipersalino, estuário do rio Pisa Sal (Galinhos, Rio Grande do Norte, Brasil). Tropical Oceanography, v. 33, n. 1, p. 39-55, 2005.

SANTOS, T. G.; BEZERRA-JÚNIOR, J. L.; COSTA; K. M. P.; FEITOSA, F. A. N. Dinâmica da biomassa fitoplanctônica e variáveis ambientais em um estuário tropical (Bacia do Pina, Recife, PE). Revista Brasileira de Engenharia de Pesca, v. 4, n. 1, p. 95-109, 2009.

SHEN, P. P.; LI, G.; HUANG, L. M.; ZHANG, J. L.; TAN, Y. H. Spatio-temporal variability of phytoplankton assemblages in the Pearl River estuary, with special reference to the influence of turbidity and temperature. Continental Shelf Research, v. 31, p. 16721681,2011

SIN, Y.; WETZEL, R. L.; ANDERSON, I. C. Seasonal variations of size-fractionated phytoplankton along the salinity gradient in the York river estuary, Virginia (USA). Journal of Plankton Research, v. 22, n. 10, p. 1945-1960, 2000.

SILVA, S. S. L.; ZICKEL, C. S.; CESTARO, L. A. Flora vascular e perfil fisionômico de uma restinga no litoral sul de Pernambuco, Brasil. Acta botânica brasílica, v. 22, n. 4, p. 1123-1135, 2008.

STRICKLAND, J.D.H; PARSONS, T.R. A practical handbook of seawater analysis. Bulletin Fisheries Research Board of Canada, v. 167, p. 1-211, 1972.

STATSOFT, Inc. Statistica: data analysis software system, version 7, 2004.

THOMPSON, P. A.; BONHAM, P. I.; SWADLING, K. M. Phytoplankton blooms in the Huon Estuary, Tasmania: top-down or bottom-up control?. Journal of Plankton Research, $v$. 30, n. 7, p. 735-753, 2008. DOI:10.1093/plankt/fbn044

UNESCO. Determination of photosynthetic pigments in sea waters. Report of SCOR/UNESCO working group 17 with meat from 4 to 6 june 1964, Paris: s.n., 1966. 69 p. (Monographys on Oceanology Methodology).

VASCONCELOS, D. M.; SANTOS, P. J. P.; LOPES, R. 2004. Distribuição espacial da meiofauna no estuário do rio Formoso, Pernambuco, Brasil. Atlântica, v. 26, n. 1, p. 4554, 2004.

VAN DER MOLEN, J. S.; PERISSINOTTO, R. Microalgal productivity in an estuarine lake during a drought cycle: The St. Lucia Estuary, South Africa. Estuarine, Coastal and Shelf Science, v. 92, p. 1-9, 2011 